\title{
SET COVER PROBLEM OF COVERAGE PLANNING IN LTE-ADVANCED RELAY NETWORKS
}

\author{
Fan-Hsun Tseng \\ National Central University \\ No. 300, Jhongda Rd., Jhongli City, Taoyuan County 32001, Taiwan \\ fanhsuntseng@ieee.org \\ Li-Der Chou \\ National Central University \\ No. 300, Jhongda Rd., Jhongli City, Taoyuan County 32001, Taiwan \\ cld@csie.ncu.edu.tw \\ Han-Chieh Chao \\ National I-Lan University/National Dong Hwa University \\ No. 1, Sec. 1, Shen-Lung Rd., I-Lan, 26047, Taiwan \\ No. 1, Sec. 2, Da Hsueh Rd., Shoufeng, Hualien 97401, Taiwan \\ hcc@niu.edu.tw \\ Wei-Jen Yu \\ National I-Lan University \\ No. 1, Sec. 1, Shen-Lung Road, I-Lan, 26047, Taiwan \\ ayumi_5420@hotmail.com
}

\begin{abstract}
Various mobile devices are developing rapidly in contemporary society, such as smart phones and tablet PCs. Users are able to acquire different multimedia services through wireless communication anytime and anywhere. However, the increased demand also gives rise to a problem of insufficient bandwidth. Therefore, a fourth generation mobile telecommunications (4G) technology was proposed and widely investigated. One of the popular technologies is Long Term Evolution Advanced (LTE-Advanced), which was proposed by the Third Generation Project Partnership (3GPP). The Evolved Node B (eNB) and Relay Node (RN) are the major components in an LTE-Advanced network. How best to deploy these two components to extend network coverage and expand performance is a vital issue. In this paper, we utilize an integer linear programming model (ILP) to formulate the coverage problem, and refer to a well-known problem called the Set
\end{abstract}


Cover problem. Then we propose a heuristic algorithm named as the Set Covering algorithm to solve it. The ultimate object is achieving the highest network coverage and capacity with the least uncovered mobile user. In the simulation result, we use MATLAB to simulate a network deployment, and evaluate the planning results. According to the simulation results, we accomplished better network capacity and a higher number of covered users.

Keywords: LTE-Advanced, Relay Technology, Network Planning, Integer Linear Programming, Set Cover Problem

\section{INTRODUCTION}

Long Term Evolution (LTE) is a mobile communication standard that was proposed by the Third Generation Project Partnership (3GPP) ${ }^{1}$, and the subsequent version is LTE-Advanced in Release $10^{2}$. It is able to satisfy the transmission data rates requirement of 1 gigabit per second on downlink and 500 megabit per second on uplink, as defined by the International Telecommunication Union (ITU). There are two major enhancements that include carrier aggregation technology and relay technology. The carrier aggregation technology permits grouping of several different component carriers into one logical channel, hence achieving a higher peak traffic channel data rate. On the other hand, relay technology is proposed to economize on construction costs by deploying RN, and improves network throughput and coverage. Since the relay technology makes a breakthrough in LTE-Advanced networks, researchers in Germany started to investigate and analyze the performance of relay development for a realistic suburban environment ${ }^{3}$. The planning of network infrastructure is a necessary step in a developing country.

In this paper, we investigate the relay technology in LTE-Advanced networks. The relay technique is considered as a cost-efficiency solution for extending coverage and enhancing throughput, as shown in Figure 1. To provide a higher cell capacity, the $\mathrm{RN}$ is deployed between the eNB and user to eliminate transmission distance. Therefore, mobile users are able to obtain a better signal-to-noise ratio (SNR) value. Additionally, the communication path bypasses the building when RN is next to the building. The problem of a shadowing effect can be solved and overcome. Furthermore, the $\mathrm{RN}$ is also deployed near a cell edge to extend the coverage of eNB, and hence the users can be served even if they are out of range. As such, transmission distance, coverage of eNB, and network capacity can be improved by deploying RNs. 


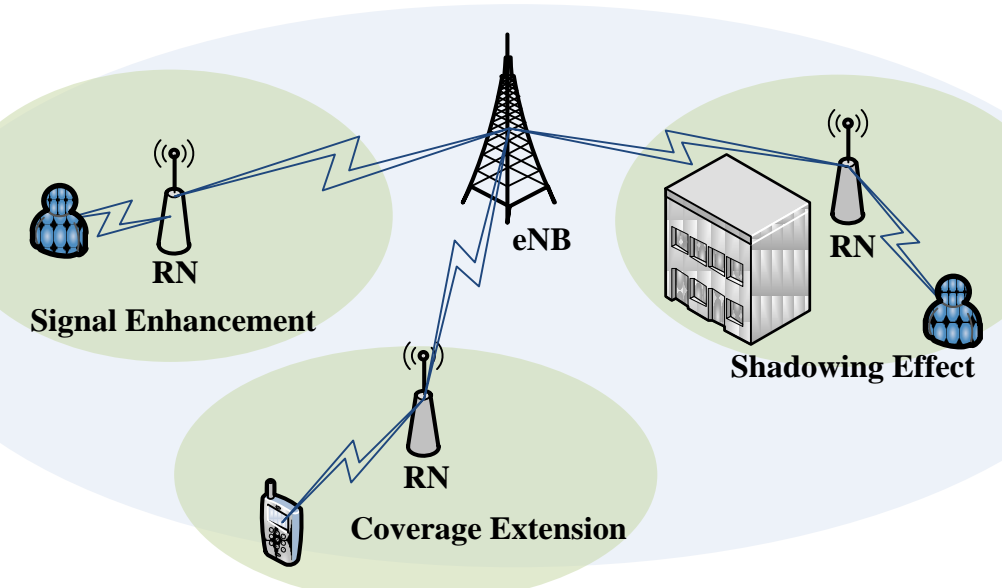

Figure 1. LTE-Advanced with relay technology scenario

The paper is organized as follows. In section II, we introduce the existing literature on an LTE-Advanced relay network, then analyze and compare different relay planning approaches. In section III, we formulate the proposed ILP model and introduce the Set Cover problem, and the proposed heuristic algorithm and the Set Covering algorithm is stated. In section IV, we firstly illustrate the planning results of the Set Covering algorithm with a planning case, then evaluate the simulation results with other approaches. Finally, conclusions and future works about this research are drawn in section $\mathrm{V}$.

\section{RELATED WORK}

No matter what kind of network, network planning is an essential process for environment construction ${ }^{4,5}$. The relay technique has been widely studied in wireless communications. Research topics include cost-efficiency, throughput enhancement and coverage extension, especially for the IEEE 802.16j and LTE-Advanced network in recent years ${ }^{6}$. The primary goal is minimizing the construction cost and maximizing the network capacity. Lu et al. ${ }^{7}$ proposed a heuristic algorithm to solve the throughput maximization relay station placement (TM-RSP) problem in IEEE 802.16j networks. Within the limitation of a budget, the proposed algorithm was deployed in relay stations (RSs) for better gains. Dejun Yang et al. $^{8}$ found the minimum number of RSs to satisfy the requests of subscribers via cooperative communication. A lower cost was achieved and the network performance was improved.

To investigate the aspect of LTE-Advanced relay placement, Gordejuela-Sanchez et al. ${ }^{9}$ formulated a mixed integer linear programming 
(MILP) model, and utilized a multi-objective Tabu search to find the Pareto front as their solution. The construction cost is the same as with our objective, but the researchers considered the site selection of eNB only, unlike including both $\mathrm{eNB}$ and $\mathrm{RN}$ in this work. Although the resource block and transmission power for uplink were considered by Majewski and Koonert ${ }^{10}$, they did not investigate RN deployment. Li et al. ${ }^{11}$ formulated a cooperative game with power allocation and position selection of the eNB. The difference between $\mathrm{Li}$ et al. ${ }^{11}$ and us is that the researchers consider eNB distribution and power assignment, while we focus on satisfying user requirements with the best network capacity and the most users. Majewski and Koonert ${ }^{12}$ proposed a scheme to optimize the cell load, which depends on the location selection of an eNB and the tilt and azimuth of an antenna. Saleh et al. ${ }^{13}$ investigated RN placement for better coverage extension and throughput between a pico eNB and RN, while we consider both eNB and $\mathrm{RN}$ and serving the most users.

The influence of site planning for a relay network was studied by Bulakci et al. ${ }^{14}$, and the signal-to-noise-ratio (SNR) and signal-to-interference-plus-noise-ratio (SINR) were also considered. This paper also considers the SINR value as in both cases in the literature. However, previous researchers only were concerned with site selection of multiple RNs within the cell range of an eNB. It is worthy to be mentioned that the proposed Set Covering algorithm constructs multiple eNBs and RNs in a LTE-Advanced network. There are also many related works about relay technology in LTE-Advanced networks. For example, some researchers concentrated on the analysis of relay performance ${ }^{15}$, and some utilized RNs to extend network coverage ${ }^{16,17}$, and some were dedicated to probe into the relay architecture for better cost efficiency ${ }^{18}$.

We have previously published similar works ${ }^{19,20,21,22}$ to this work. We proposed a tree algorithm ${ }^{19}$ for increasing reliability in IEEE 802.16j mobile multi-hop relay (MMR) networks. Then we proposed a Supergraph Tree (S-Tree) algorithm ${ }^{20}$ to minimize construction costs for IEEE 802.16j MMR networks. The S-Tree algorithm utilized the relay station to connect disconnected subgraphs and constructed the MMR networks easily. The Interference-Aware Tree (IA-Tree) algorithm ${ }^{22}$ was proposed to solve the interference problem between two neighbor BSs, and provided the better service quality. However, these proposed algorithms were designed for a MMR network, which is different from the two-hop relaying limitation of a relay network in LTE-Advanced. Therefore, we firstly investigated the characteristics of two-hop relaying and offered proposals in a prior study ${ }^{21}$. We found that the hop count limitation definitely influences the results on construction cost, served user and network capacity. In this paper, we propose a Set Covering algorithm based on a Set Cover problem, and 
enhanced network performance and coverage with definitely acceptable construction costs.

\section{PROBLEM DEFINITION AND PROPOSED ALGORITHM}

In this section, we formulate the coverage problem of an LTE-Advanced network with an ILP model. Then the well-known Set Cover problem is introduced and the design concept of the proposed Set Covering algorithm is explained. There are two types of node in planning work, one is the candidate position (CP) for deploying the eNB and RN, and the other is the location of user equipment (UE). The ultimate goal is to determine the eNB and RN numbers with the highest network capacity and the most numbers of covered UEs.

\subsection{Problem Definition}

We introduce the problem definition herein. Let a set of vertices as $V$ and a set of edges between vertices as $E$. Given an undirected graph $G=(V, E)$, where $V=V_{1} \cup V_{2}$ and $E=E_{1} \cup E_{2}$. Then, it can be known that $V_{1} \cap V_{2}=\varnothing$ and $E_{1} \cap E_{2}=\varnothing$. Assume that a set of $\mathrm{CP} V_{1}=$ $\left\{z_{1}, \ldots, z_{k}\right\}$ with $\left|V_{1}\right|=k$, and a set of UE $V_{2}=\left\{q_{1}, \ldots, q_{s}\right\}$ with $\left|V_{2}\right|=s$. If an eNB (or RN) is placed on the location corresponding to $z_{i}$, then $z_{i}=c_{e N B}\left(\right.$ or $\left.z_{i}=c_{R N}\right)$. If there is no instrument placed on the location corresponding to $z_{i}$, then $z_{i}=0$. That is, $z_{i} \in\left\{0, c_{e N B}, c_{R N}\right\}$.

Herein, we formulate the coverage problem with ILP model for LTE-Advanced relay networks. Firstly, we consider the available links within a CP $i$ and a CP $j$, and the available links are defined as $x_{i, j}$, let

$$
x_{i, j}=\left\{\begin{array}{l}
1, \text { if }\left(z_{i}, z_{j}\right) \in E_{1} \text { and } z_{i} \cdot z_{j} \neq 0 \\
0, \text { otherwise }
\end{array}\right.
$$

This requirement of tree structure is considered in our model, where it can be represented as

$$
\sum_{e \in E(S)} x_{e} \leq|S|-1,
$$

where $S \subseteq V_{1}$ and $E(S)=\left\{(i, j) \in E_{1} \mid i, j \in S\right\}$. We define a parameter $a_{i, j}$ to represent the coverage relation between an UE $i$ and a CP $j$, where

$$
a_{i, j}=\left\{\begin{array}{l}
1, \text { if }\left(q_{i}, z_{j}\right) \in E_{2} \\
0, \text { if }\left(q_{i}, z_{j}\right) \notin E_{2}
\end{array}\right.
$$


For the functionality of an UE, it should be served by at least one eNB or RN, hence the condition $\max _{i} a_{i, j} Z_{j} \geq 1$ should be satisfied. The signal quality $w_{i}$ of an itemize UE $i$ is defined as

$$
w_{i}=\left\{\begin{array}{cl}
w_{B S}, & \text { if } z_{i}=c_{e N B} \\
w_{R S}, & \text { if } z_{i}=c_{R S} \\
0, & \text { if } z_{i}=0
\end{array}\right.
$$

Let $\lambda_{i, j}=a_{i, j} q_{i} w_{j}$. The utility of each UE is defined as $u_{i}=\max _{j} \lambda_{i, j}$. In addition, we limit the depth of routing tree within $l$, and cope with this requirement, assume

$$
y_{i, j}=\left\{\begin{array}{l}
1, \text { if }\left(q_{i}, z_{j}\right) \in E_{1}^{l} \\
0, \text { if }\left(q_{i}, z_{j}\right) \notin E_{1}^{l}
\end{array}\right.
$$

Then, let the maximum depth $l$ of routing tree $\eta_{i}=\max _{j} y_{i, j}$. To comply with the two hop limitation, we define the routing tree $\eta_{i}$ as bigger than 1 hop and less than 2 hops. The ILP model for coverage problem in LTE-Advanced relay network is defined as follows.

Minimize

subject to

$$
\sum_{i=1}^{k} z_{i} \leq \sigma,
$$

$\sum_{e \in E(S)} x_{e} \leq|S|-1$,

$\sum_{i=1}^{S} \frac{u_{i}}{s} \geq \delta$,

$1 \leq \eta_{i} \leq 2, \forall i$

$\max _{j} a_{i, j} z_{j} \geq 1$

\subsection{Set Cover Problem and Set Covering Algorithm}

The Set Cover problem is a well-known problem in set theory, which has been applied to many research fields. A brief introduction is described as follows. By definition, given an universal set $U$ with $m$ elements as $U=\{1,2, \ldots, m\}$, and finds $n$ sets whose union comprises the universal set $U$. Thereby, the Set Cover problem identifies the smallest numbers of set whose union still containing all elements in the universe. For instance, given the universal set $U=\{1,2,3,4,5\}$ with five elements, and a set $S=$ $\{\{1,2,3\},\{2,4\},\{3,4\},\{4,5\}\}$ with five subsets. It's obvious that the union of all given subsets in $S$ contains all elements in the universal set $U$. Therefore, we can cover the universal set with two subsets that are $\{1,2,3\}$ and $\{4,5\}$ as the smallest set numbers. As the above statement, we approach the concept of the Set Cover problem to construct the relay 
network. Firstly, we assume that the universal set is all UEs in network, and each subset is regarded as a $\mathrm{CP}$ that means an $\mathrm{eNB}$ or RN covering the UEs. Let $S_{1}=\{1,2,3\}, S_{1}$ represents an eNB or RN which covers the $U E_{1}$, $U E_{2}$ and $U E_{3}$. We would like to find the smallest number of subsets that implies the least number of constructed sites. Therefore, we will find the most number of covered UEs with the lowest construction cost.

Based on the Set Cover problem, we design a heuristic algorithm named Set Covering algorithm to solve the coverage problem in LTE-Advanced networks. The principle steps are stated as follows. First of all, we examine the user who is served by the least CPs, and select the CP that serves the most UEs and deploy an eNB at this site. In this way, the UEs can be covered and served by deploying this eNB, and the deployed eNB serves the most numbers of UEs. After that, we select some specific CPs to deploy RNs, which serve the UEs within the coverage range of the deployed eNB. Finally, repeats the above steps until all of the UEs are served. The difference between previous methods with ours is the consideration of the deployment order. In previous algorithms, the deployment priority of RN is higher than eNB thus the performance of capacity is worse. The Set Covering algorithm is contrary to previous algorithms, hence a better network performance and more UEs can be achieved and served.

The Set Covering algorithm is shown as Table 1. Denote $X$ and $Y$ as the subsets of vertices. Then, $f_{d}(G, X, Y)$ is a descendant ordered list $S$, which inducts the elements in $X$ in terms of the adjacent number of nodes in $Y$ based on the given graph $G$, and the ascendant ordered list $S^{\prime}$ vice versa. The sequence of vertices can be arbitrary if there are some nodes in the same degree. Owing to $S$ is an ordered list, the $i$-th vertex in $S$ is denoted as $S[i]$. Lines 2 to 6 are used to find CP for deploying eNB, that serves the most UEs. Lines 10 to 15 select the CPs to deploy RNs until there are no uncovered UEs. In addition, lines 16 to 19 guarantee that the required utility rate can be achieved, otherwise an eNB is deployed to replace the RN. Finally, the property that the hop count limitation between the eNB and RN should be satisfied and checked with two-hop relaying is in lines 21 to 25 .

Herein, the computational complexity of proposed algorithm is calculated and analyzed. The performance of the Set Covering algorithm is dominated by lines 2 to 15 . The Set Covering algorithm is similar to the Tree and S-Tree algorithm, as it sorts $\mathrm{O}(\mathrm{n})$ elements in each iteration, hence the $O(n \operatorname{logn})$ time complexity is needed. The difference between previous algorithms and the Set Covering algorithm is the order of element $X$ and element $Y$ in the descendant ordered list $S$. A brief summary, the previous algorithms are site-oriented and it is UE-oriented in the Set Covering algorithm. 
Table 1. Set Covering algorithm

Algorithm: Set Covering Algorithm $(G, z, \delta, l)$

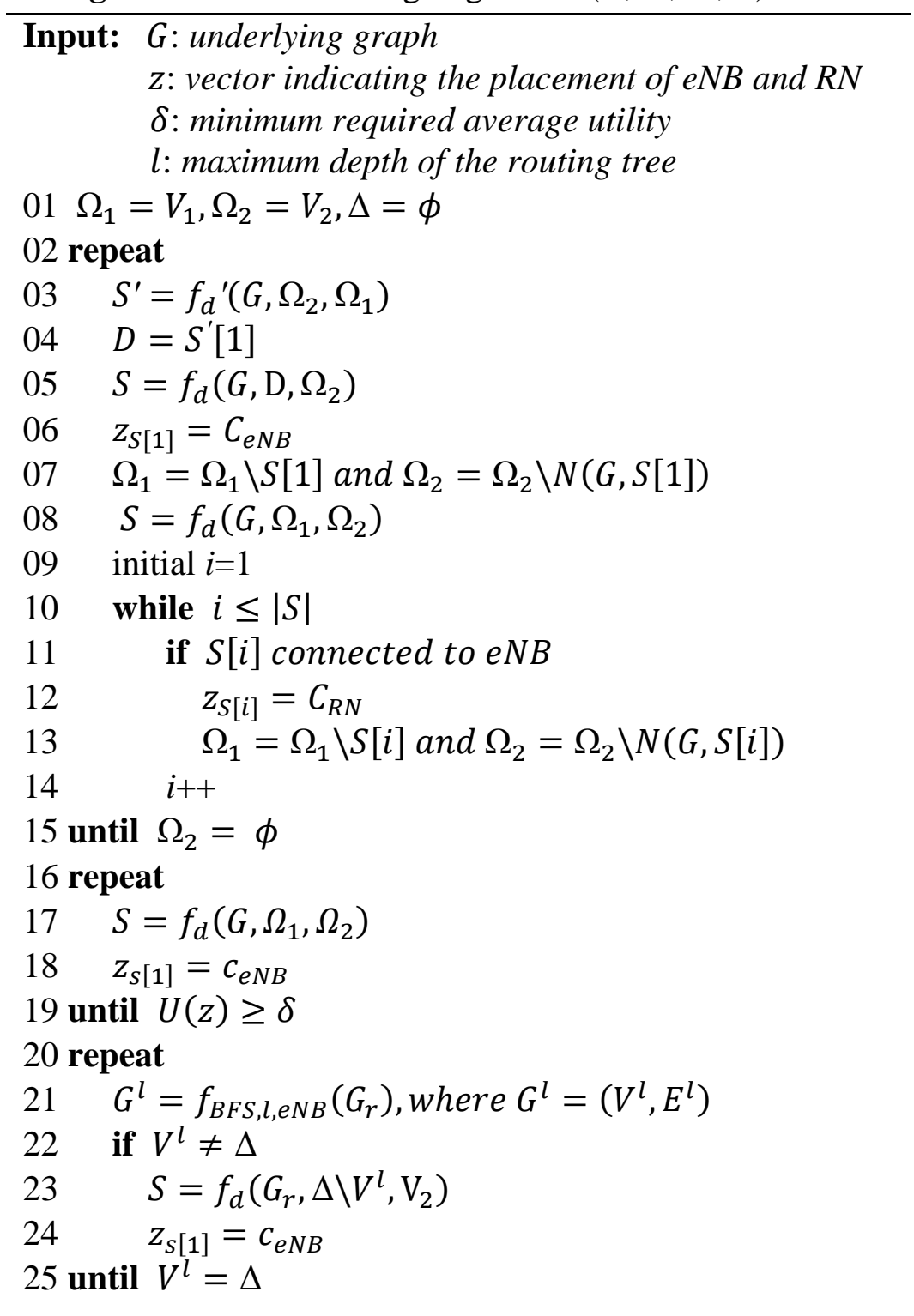

\subsection{Evaluation of Network Planning}

In this subsection, we explain how to evaluate the planning results. As mentioned, we focused on network coverage, which implies the least uncovered UEs, and achieving better network capacity. The detail definitions of measurement metrics are defined as follows. 
1. Construction cost: we simply define the construction cost as related to the deployment cost of eNBs and RNs, rather than considering recurring operational expenditure (i.e. Operation \& Maintenance, site rent etc.)23. The construction cost of an eNB is 10 unit costs, and the $\mathrm{RN}$ is 2.5 units.

2. Communication interference: we consider that the UEs may receive multiple signals from various eNBs and RNs, hence there exists communication interference. For example, if an UE receives three signal sources, then one of the signals is the major signal and others are regarded as interference. The $I_{s}$ represents the receive interference of UEs, let

$$
I_{s}=\sum_{i=1}^{k} \frac{{\text { Trans } \text { Power }_{k}}_{\text {Path } \text { Loss }_{k}}}{\text { Pat }}
$$

The Trans Power $_{k}$ is the transmission power of signal from CP $k$, and the Path Loss $k$ is the path loss between the UEs and CP $k$.

3. Uncovered users: we focus on the coverage of the planning network, thus the number of uncovered users is calculated. Each UE has a SINR value and represents the communication quality. If the communication quality is too low to communicate, we define it as belonging to an uncovered user. The free space propagation $\operatorname{model}^{7}$ is utilized to calculate the SINR value, that

$$
\operatorname{SINR}=10 * \log _{10}\left(\frac{P_{t}}{I} *\left(\frac{c}{4 \pi f d}\right)^{2}\right)
$$

The $P_{t}$ is the transmission power from the signal source, $I$ represents the interference, $f$ is the frequency band, $c$ is the speed of light, and $d$ is the distance between site and user.

4. Capacity of users: since we obtained the SINR value of UEs in equation (7). The Shannon capacity theory is used to calculate the capacity of UEs. It is shown as follows:

$$
C=B \log _{2}(1+\operatorname{SINR})
$$

Where $C$ is the channel capacity in bits per second, and $B$ is the bandwidth of the channel in hertz.

\section{EXPERIMENT RESULTS}

The simulation was done with MATLAB ${ }^{24}$, and the experimental computer had a CPU with two cores, $1.7 \mathrm{GHz}$ and $3 \mathrm{~GB}$ RAM. The 
simulation was simulated with five different network scenarios (randomly distribute), and calculated the average to avoid a singular state. The major simulation parameters are shown in Table 2.

Table 2. Simulation parameters

\begin{tabular}{cc}
\hline Variables/parameters & Value \\
\hline Topology size & $25 \times 25(\mathrm{KM})$ \\
Number of CPs & 50 \\
Number of UEs & 300 \\
Radius of eNB & $3400(\mathrm{M})$ \\
Radius of RN & $1000(\mathrm{M})$ \\
Deployment cost of eNB & $10($ units $)$ \\
Deployment cost of RN & $2.5($ units $)$ \\
Transmission power of eNB & $47(\mathrm{dBm})$ \\
Transmission power of RN & $43(\mathrm{dBm})$ \\
\hline
\end{tabular}

\subsection{Development Case}

In this subsection, we illustrate the Set Covering algorithm with a deployment case. In the planning case, there are $50 \mathrm{CPs}$ marked as blue circles, and 300 UEs are labeled as red stars within the 25 kilometers multiplies a 25 kilometers network. The random network topology is shown as Figure 2.

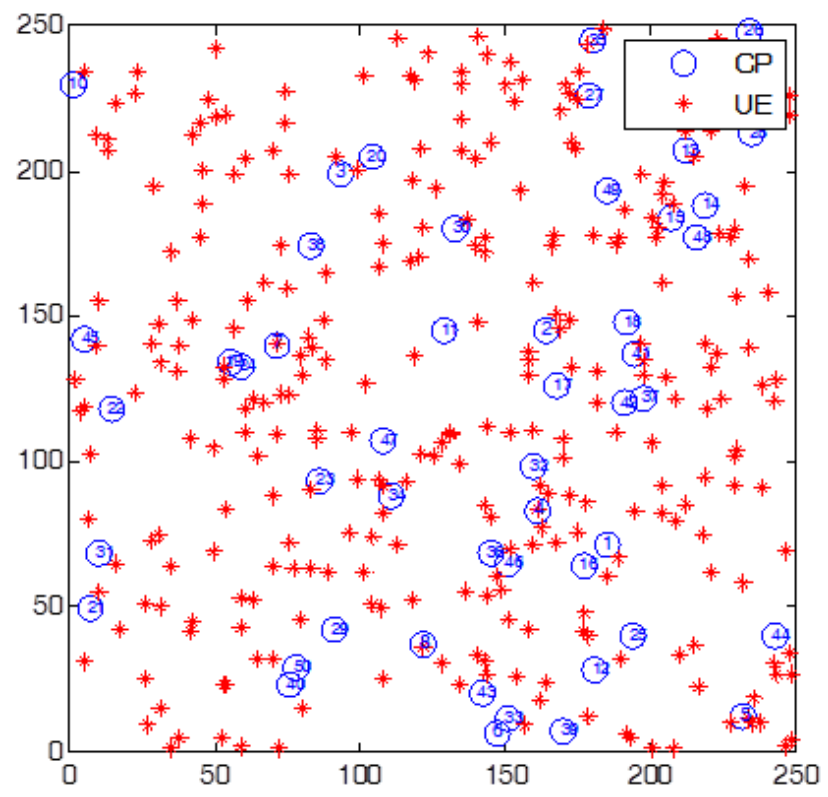

Figure 2. Network topology 
The planning result of the Set Covering algorithm is shown in Figure 3. The dark blue solid points represent the CPs that were deployed with eNBs, and the light blue hollow points are the RNs. The blue line represents the relay link from eNB to RN, and the red dotted line is the connection of UE. All nodes were randomly distributed in our planning case.

The main object was to cover and serve the most UEs. Because the proposed Set Covering algorithm is based on the Set Cover problem, the UEs covered by the least number of CPs should be served first. Then an eNB should be placed on this CP which covers the most UEs. Finally, we repeatedly deploy RNs to connect with as many eNBs as possible.

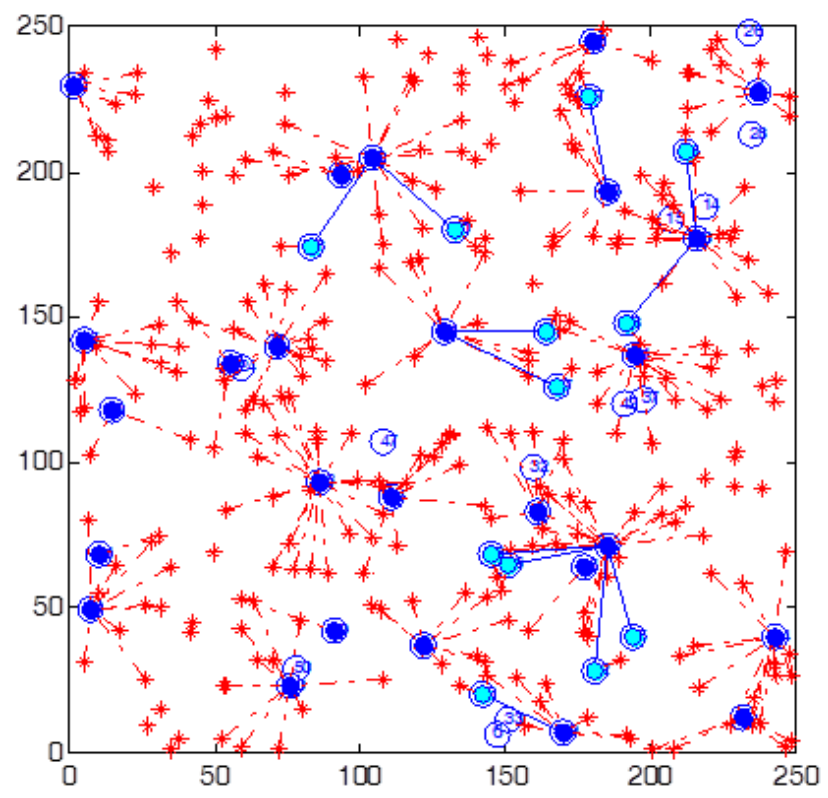

Figure 3. Deployment result of Set Covering algorithm

Figure 3 shows that there are $26 \mathrm{eNBs}$ and $12 \mathrm{RNs}$, and the total construction cost is summed up as 272 unit costs. According to the 3.3 subsection, we evaluated the performance and results of the planned network. There were 41 uncovered users, and the total capacities of the UEs were 7519.66 bits per second.

\subsection{Results and Analysis}

In the simulation results, we will show the comparisons of different factors, including the network planning time, construction cost, number of uncovered users and network capacity. The compared algorithms are Tree ${ }^{19}$, Supergraph-Tree ${ }^{21}$ and Interference-Aware Tree ${ }^{22}$ algorithm. The Tree algorithm was designed for the multi-hop relay network in IEEE 802.16j, 
and the relay stations deployed by S-Tree algorithm were chosen from the unselected candidate positions. The IA-Tree algorithm eliminated the interference between two nearby base stations. These three algorithms were compared with the proposed algorithm in this paper.

Firstly, the computation efficiency of all algorithms is shown as Figure 4. We increased the number of CPs and calculated the planning time. As Figure 4 shows, the planning time of the four algorithms is linear and less than two seconds. However, the proposed algorithm is slightly longer than previous algorithms. This is because the sorting order of the Set Covering algorithm is different with previous algorithms. We aim to provide a better network capacity for users and serve more users, and the descending order list $S^{\prime}$ of Set Covering algorithm is UEs $\Omega_{2}$ links to CPs $\Omega_{1}$ rather than the CPs $\Omega_{1}$ covers UEs $\Omega_{2}$. Meanwhile, the number of UEs is always higher than the numbers of CPs. Therefore, the Set Covering algorithm spends more planning time when it searches recursively to cover all UEs. Although the planning time proposed by the Set Covering algorithm is slightly longer than others, the planning process was still completed in two seconds. In other words, the proposed algorithm and previous algorithms are suitable for a large-scale planning area and a complicated planning environment.

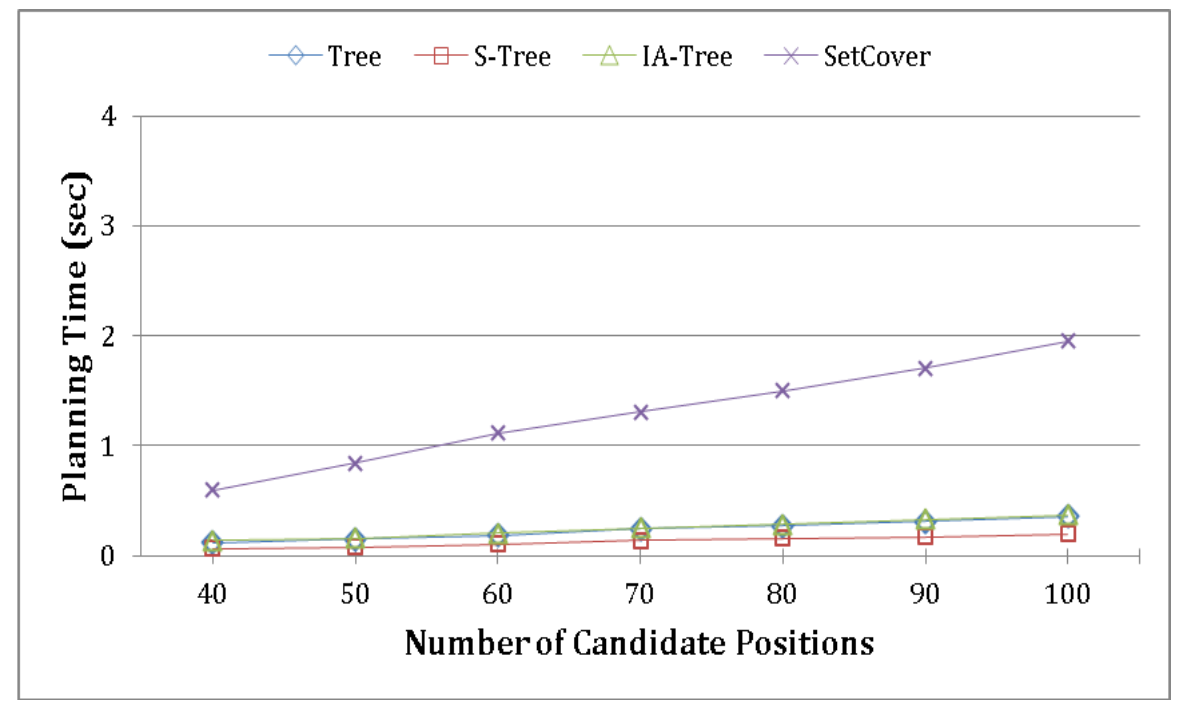

Figure 4. Calculation time with varying the number of CPs

The construction cost in the different network topologies is shown in Figure 5. The simulation parameters are the same as with Table 2, and we calculated the average deployment cost of the four algorithms. The Tree algorithm deploys eNBs on most parts of the CPs, and only seldom places 
RNs. Therefore, the average construction cost of the Tree algorithm is the highest. The construction costs of the S-Tree and IA-Tree algorithm are lower than the Tree and Set Covering algorithms. The Tree algorithm was designed to minimize construction costs by principally placing RNs, hence its construction cost is lower. On the other hand, because the communication interference between eNBs is considered in the IA-Tree algorithm, nearby eNBs are replaced with RNs. Although the construction cost of the IA-Tree algorithm is the lowest, it also results in a higher number of uncovered users than the other algorithms. Because the Set Covering algorithm was designed to cover as many UEs as possible, it has a higher construction cost, but this is still lower than the Tree algorithm.

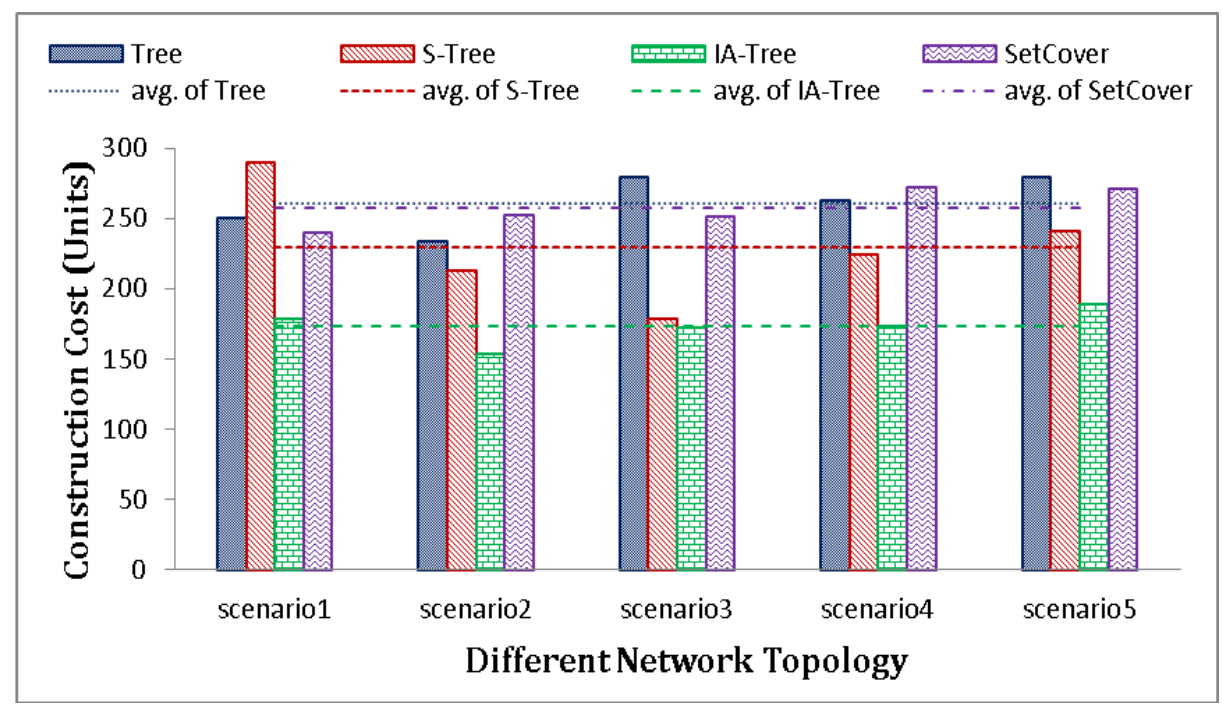

Figure 5. Construction costs in five different network topologies

The number of uncovered users in different network topologies is shown as Figure 6 . Both the S-Tree and IA-Tree algorithm were designed to deploy RNs, so the unserviceable UEs are higher than in the Tree and Set Covering algorithms. Although the difference in construction costs between the Tree and Set Covering algorithm is slight, the uncovered UEs of the proposed algorithm is much lower than the Tree algorithm. According to the results, it is obvious that the proposed Set Covering algorithm achieves better network coverage. In other words, there are more UEs that can be satisfied in the planning result of the proposed Set Covering algorithm. 


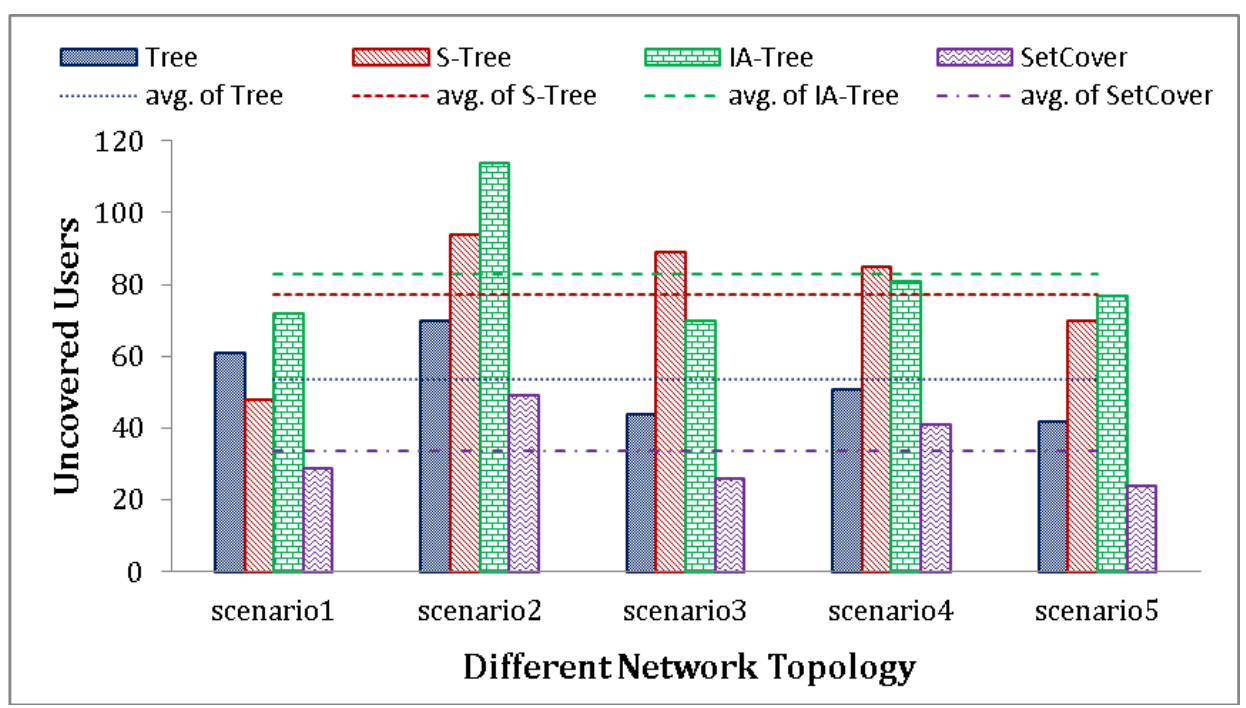

Figure 6. Number of uncovered users in five different network topologies

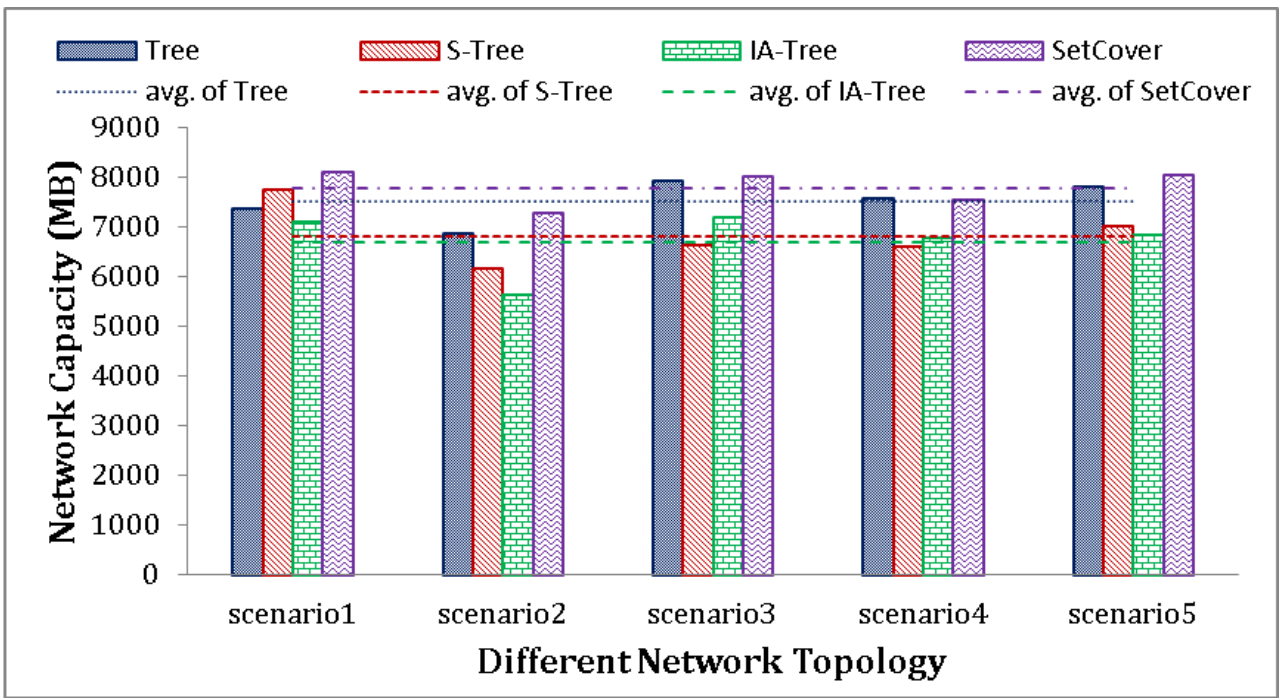

Figure 7. Network capacity in five different network topologies

We evaluated network capacity based on equation 8 in subsection 3.3, and compared the four algorithms in five different network topologies, with the results shown in Figure 7. It should be noted here that the network capacity is summed of all covered UEs in eNBs and RNs. Although the construction costs of S-Tree and IA-Tree is low, their uncovered UEs and network capacity is not ideal. Unlike the S-Tree and IA-Tree algorithm, the Tree and Set Covering algorithms achieve a higher network capacity. Furthermore, the proposed Set Covering algorithm accomplishes better 
network capacity than the Tree algorithm with a lower construction cost and fewer uncovered UEs.

\section{CONCLUSIONS AND FUTURE WORK}

For large-scale network planning, cost efficiency is a vital issue. In this paper, we formulated a coverage problem based on the well-known Set Cover problem, and proposed a Set Covering algorithm to achieve better network coverage and performance for LTE-Advanced relay networks. We offer three major contributions thru this research: 1) a network of multiple eNBs and RNs is considered and constructed; 2) the linear planning time of the proposed algorithm is accomplished; and 3) the best network coverage and capacity of four algorithms is achieved and presented. According to the simulation results, the proposed Set Covering algorithm is suitable for planning that offers better network quality in a crowded city.

In the future work, we will consider how to find a solution to the inevitable trade-off between factors such as construction cost, communication quality and covered user. A multi-objective optimization problem will be considered and defined, and an evolutionary algorithm will also be designed and proposed. We expect that the results will improve planning for LTE-Advanced relay networks.

\section{ACKNOWLEDGE}

This research was partly funded by the IPv6 Upgrade and Promotion Program (1/4) under grants 102J2207001 and the National Science Council (NSC) of the Taiwan under grants NSC 101-2221-E-197-008-MY3.

\section{REFERENCES}

[1] S. Parkvall, E. Dahlman, A. Furuskar, Y. Jading, M. Olsson, S. Wanstedt, and K. Zangi, LTE-Advanced - evolving LTE towards IMT-Advanced. In E. Aubrey (Ed.), Proceedings of IEEE 68th Vehicular Technology Conference (p1-5). Calgary, BC: IEEE Press, 2008. http://dx.doi.org/10.1109/VETECF.2008.313.

[2] M. Iwamura, K. Etemad, M.-H. Fong, R. Nory, and R. Love, Carrier aggregation framework in 3GPP LTE-advanced [WiMAX/LTE Update]. IEEE Communications Magazine, 48(8), p60-67, 2010. http://dx.doi.org/10.1109/MCOM.2010.5534588.

[3] C. Coletti, P. Mogensen, and R. Irmer, Performance analysis of relays in LTE for a realistic suburban deployment scenario. In P. Nagy and R. Vida (Eds.), Proceedings of IEEE 73rd Vehicular Technology Conference (p1-5). Budapest: IEEE Press, 2011. http://dx.doi.org/10.1109/VETECS.2011.5956245. 
[4] H. Liang, I.Z. Kovacs, P. Mogensen, O. Klein, and W. Stormer, Optimal new site deployment algorithm for heterogeneous cellular networks. In S. Young (Ed.), Proceedings of IEEE Vehicular Technology Conference (p1-5). San Francisco, CA: IEEE Press, 2011. http://dx.doi.org/10.1109/VETECF.2011.6092846.

[5] Y. Xie, Planning of 3G-oriented transmission network. In Z. Li (Ed.), Proceedings of International Conference on Consumer Electronics. Communications and Networks (p5122-5125). XianNing, China: IEEE Press, 2011. http://dx.doi.org/10.1109/CECNET.2011.5768243.

[6] Y. Yang, H, Hu, J. Xu, and G. Mao, Relay technologies for WiMax and LTE-Advanced mobile systems. IEEE Communications Magazine, $47(10)$, p101-105,

2009. http://dx.doi.org/10.1109/MCOM.2009.5273815.

[7] H.-C. Lu, W. Liao, and F.Y.-S. Lin, Relay station placement strategy in IEEE 802.16j WiMAX networks. IEEE Transactions on Communications, 59(1), p151-158, 2011. http://dx.doi.org/10.1109/TCOMM.2010.110310.090558.

[8] D. Yang, X. Fang, G. Xue, and J. Tang, Relay station placement for cooperative communications in WiMAX networks. In M. Karimi, S. Makki, and K. Levitt (Ed.), Proceedings of IEEE Global Telecommunications Conference (p1-5). Miami, FL: IEEE Press, 2010. http://dx.doi.org/10.1109/GLOCOM.2010.5683329.

[9] G.-S. Fernando, and Z. Jie, LTE access network planning and optimization: A service-oriented and technology-specific perspective. In B. Kozbe (Ed.), Proceedings of IEEE Global Telecommunications Conference (p1-5). Honolulu, Hawaii: IEEE Press, 2009. http://dx.doi.org/10.1109/GLOCOM.2009.5425478.

[10] K. Majewski, and M. Koonert, Analytic uplink cell load approximation for planning fractional power control in LTE networks. Telecommunication Systems, 52(2), p 1081-1090, 2013. http://dx.doi.org/10.1007/s11235-011-9614-1.

[11] Z. Li, and S. Li, LTE network planning based on game theory. In T. Ma (Ed.), Proceedings of International Conference on Computer Science and Service System (p3963-3966). Nanjing, China: IEEE Press, 2011. http://dx.doi.org/10.1109/CSSS.2011.5973956.

[12] K. Majewski, and M. Koonert, Conservative cell load approximation for radio networks with shannon channels and its application to LTE network planning. In J. Guerrero (Ed.), Proceedings of Sixth Advanced International Conference on Telecommunications (p219-225). Barcelona, Spain: IEEE Press, 2010. http://dx.doi.org/10.1109/AICT.2010.9.

[13] A.B. Saleh, S. Redana, B. Raaf, and J. Hamalainen, Comparison of relay and pico eNB deployments in LTE-Advanced. In J. Miller (Ed.), 
Proceedings of IEEE 70th Vehicular Technology Conference (p1-5). Anchorage, Alaska, USA: IEEE Press, 2009. http://dx.doi.org/10.1109/VETECF.2009.5378828.

[14] O. Bulakci, S. Redana, B. Raaf, and J. Hamalainen, Performance enhancement in LTE-Advanced relay networks via relay site planning. In C.-P. Li and Y.-W. Peter Hong (Eds.), Proceedings of IEEE 71st Vehicular Technology Conference (p1-5). Taipei, Taiwan: IEEE Press, 2010. http://dx.doi.org/10.1109/VETECS.2010.5493978.

[15] L. Rong, S.E. Elayoubi, and O.B. Haddada, Impact of relays on LTE-Advanced performance. In R. Peplow (Ed.), Proceedings of IEEE International Conference on Communications (p1-6). Cape Town, South Africa: IEEE 2010. http://dx.doi.org/10.1109/ICC.2010.5502349.

[16] R. Irmer, and F. Diehm, On coverage and capacity of relaying in LTE-Advanced in example deployments. In P. Chatzimisios and V. Kueh (Eds.), Proceedings of IEEE International Symposium on Personal, Indoor and Mobile Radio Communications (p1-5). Cannes, France: IEEE Press, 2008. http://dx.doi.org/10.1109/PIMRC.2008.4699896.

[17] T. Beniero, S. Redana, J. Hamalainen, and B. Raaf, Effect of relaying on coverage in 3GPP LTE-Advanced. In G. Femenias (Ed.), Proceedings of IEEE Vehicular Technology Conference (p1-5). Barcelona, Spain: IEEE Press, 2009. http://dx.doi.org/10.1109/VETECS.2009.5073520.

[18] X. Huang, F. Ulupinar, P. Agashe, D. Ho, and G. Bao, LTE relay architecture and its upper layer solutions. In M. Karimi, S. Makki, and K. Levitt (Eds.), Proceedings of IEEE Global Telecommunications Conference (p1-6). Miami, Florida: IEEE Press, 2010. http://dx.doi.org/10.1109/GLOCOM.2010.5683640.

[19] C.-Y. Chen, Y.-S. Liang, C.-M. Yu, C.-H. Ho, and S.-Y. Kuo, Increasing reliability for IEEE $802.16 \mathrm{j}$ mobile multi-hop relay networks planning. Paper presented at the IEEE 15th Pacific Rim International Symposium on Dependable Computing, Shanghai, China, November 16-18, 2009.

[20] F.-H. Tseng, C.-Y. Chen, and H.-C. Chao, Minimizing construction cost for IEEE 802.16j multi-hop relay networks. Paper presented at the International Conference on Pervasive Computing and Application, Maribor, Slovenia, December 1-3, 2010.

[21] F.-H. Tseng, C.-Y. Chen, L.-D. Chou, T.-Y. Wu, and H.-C. Chao, A study on coverage problem of network planning in LTE-Advanced relay networks. In A. Koyama, S. Huang, N. Bessis and J. Li (Eds.), Proceedings of IEEE 26th International Conference on Advanced 
Information Networking and Applications (p944-950). Fukuoka, Japan: IEEE Press, 2012. http://dx.doi.org/10.1109/AINA.2012.82.

[22] C.-Y. Chen, F.-H. Tseng, C.-F. Lai, and H.-C. Chao, Network planning for mobile multi-hop relay networks. Wireless Communications and Mobile Computing, 2013. Retrieved on July 10, 2013, from http://onlinelibrary.wiley.com/doi/10.1002/wcm.2396/full. http://dx.doi.org/10.1002/wcm.2396.

[23] E. Lang, S. Redana, and B. Raaf, Business impact of relay deployment for coverage extension in 3GPP LTE-Advanced. In P. Rost (Ed.), Proceedings of IEEE International Conference on Communications Workshops (p1-5). Dresden, Germany: IEEE Press, 2009. http://dx.doi.org/10.1109/ICCW.2009.5208000.

[24] MATLAB. Retrieved on May 31, 2013, from http://www.mathworks.com/products/matlab/. 